\title{
STABILITY LIMITS FOR A CLAMPED SPHERICAL SHELL SEGMENT UNDER UNIFORM PRESSURE*
}

\author{
BY \\ ROBERT R. ARCHER \\ Massachusetts Institute of Technology
}

\begin{abstract}
Summary. An integration procedure for the differential equations for the finite deflections of clamped shallow spherical shells under uniform pressure is developed. Stability limits for the clamped shell are obtained for a range of the central height to thickness ratio from about 1 to 35 . This serves to correct and extend previously known stability limits for this problem.
\end{abstract}

1. Introduction. The existing literature on the subject of spherical shells allowing finite deflections under uniform radial pressure or point load at the apex divides into two parts. One part represented by the work of von Karman and Tsien $[12,13,15]$;** Friedrichs [4]; Yoshimura and Uemura [14, 16]; Mushtari and Surkin [6]; and Feodosev [3] involves a determination of buckling pressures by means of a minimization of a potential energy expression for the shell with respect to a special class of deflection functions. Because of the rather special form of the assumed deflections in these papers, it is difficult to compare these results with integrations of the non-linear equations which as is noted in [8] can be derived as the Euler equations of the variational problem to minimize the potential energy of the shell; and therefore whose integrals correspond to a minimization with respect to a completely general class of deflection functions.

The other part represented by the work of Biezeno [2], Kaplan and Fung [5], and Simons [9] is based on integrations of non-linear differential equations corresponding to those which are used in this paper for shallow spherical shells. However, since Biezeno integrated the equations after assuming special forms for the non-linear terms in the differential equations, it is difficult to decide what influence this has on the results.

Kaplan and Fung are able to get integrals of the non-linear equations, but unfortunately they are able to determine buckling pressures, stresses, and deflections only for very low shells where the deflection shapes are of a simple type. In this range, their results are correct as far as they have gone in the perturbation of the non-linear equations, but appreciable corrections are to be found in the higher perturbations even in this range.

Simons generalizes the power series method given by Way for flat plates (see [11], p. 338) to shallow spherical shells. Numerical results when compared with those of Kaplan and Fung for the clamped shell are found to differ considerably owing to the retention of only a few terms in the power series solution.

In this paper (and in [2, 5, 9]), the so-called "classical criterion" of buckling as distinguished from the "energy criterion" developed by von Karman and Tsien is applied to interpret the buckling phenomenon. In the "classical criterion", it is assumed that a given state of equllibrium becomes unstable when there are equilibrium positions

*Received October 26, 1956. The material in this paper was submitted as part of a thesis by the author to the Massachusetts Institute of Technology, May 1956, in partial fulfillment of the requirements for the degree of Doctor of Philosophy.

${ }^{* *}$ Numbers in brackets refer to references at end of paper. 
infinitesimally near to that state of equilibrium for the same external load. Thus, it is a question of obtaining the pressure-deflection relations for a given problem and properly interpreting the buckling pressure according to the above criterion. It is found that the center deflection to pressure relation used in $[2,5,9]$ to interpret buckling must be generalized by interpreting buckling from a maximum deflection (in general, away from the center) to pressure relation in order to reveal the buckling in the cases where the deflection modes get more involved.

It might be noted here that problems of finite axi-symmetric deflections of flat plates are included as a limiting case of the shallow shell, and thus the methods given in this paper carry over to these problems.

With a view to the application of high speed digital computing equipment, the basic approach in this paper has been to reduce the integration of the non-linear differential equations to the problem of solutions of algebraic equations by means of suitable sets of functions for the various cases. Thus, the rapidly increasing store of methods for applying computers to solving algebraic equations can be brought to bear on these problems.

2. Equations for shallow spherical shells. The equations for the finite deflections of shallow spherical shells under uniform radial pressure which form the basis of this analysis are derived by Reissner in [8] and are listed here for reference:

$$
\begin{gathered}
D / a\left(\beta^{\prime \prime}+\beta^{\prime} / \xi-\beta / \xi^{2}\right)=-\Psi+\frac{1}{2} \rho a^{2} \xi+\Psi \beta / \xi, \\
1 / E h a\left(\Psi^{\prime \prime}+\Psi^{\prime} / \xi-\Psi / \xi^{2}\right)=\beta-\frac{1}{2} \beta^{2} / \xi, \\
a V=\frac{1}{2} \rho a^{2} \xi, \quad a Q=-\Psi+\frac{1}{2} \rho a^{2} \xi+\Psi \beta / \xi, \\
a N_{\xi}=\Psi / \xi, \quad a N_{\theta}=\Psi^{\prime}, \\
a M_{\xi}=D\left(\beta^{\prime}+\nu \beta / \xi\right), \quad a M_{\theta}=D\left(\beta / \xi+\nu \beta^{\prime}\right),
\end{gathered}
$$

Fig. 1. Element of the shell showing stress resultants and couples. 
The equations of the middle surface of the spherical shell in its undeformed state are taken in the form

$$
r_{0}=a \sin \xi \quad z_{0}=-a \cos \xi \quad 0 \leq \xi \leq \xi_{0},
$$

where $\xi_{0}$ is to represent one-half the opening angle of the shell. The components of surface loading for uniform radial pressure take the form

$$
p_{h}=\rho \sin \phi \quad p_{v}=-\rho \cos \phi
$$

Equations (1) and (2) are the result of restricting attention to shallow shells where $\xi_{\text {. }} \ll \pi / 2$.

The following non-dimensional form of the variables will be used

$$
\begin{gathered}
\beta^{*}=\beta / \xi_{\circ}, \quad \psi^{*}=\Psi / E h \xi_{e} / m^{2}, \\
p=-\rho / \rho_{c r}, \quad x=\xi / \xi_{\odot}, \\
\lambda^{2}=\xi_{\bullet}^{2} m^{2} a / h, \quad m^{4}=12\left(1-\nu^{2}\right),
\end{gathered}
$$

where $\rho_{c r}$ is the minimum buckling pressure for the corresponding complete sphere from the linear theory $\left(\rho_{c r}=4 E h^{2} / m^{2} a^{2}\right.$, see [10]). Using the non-dimensional variables (1) becomes

where

$$
\begin{gathered}
1 / \lambda^{2} L^{*} \beta^{*}+\psi^{*}=-2 p x+\psi^{*} \beta^{*} / x \\
1 / \lambda^{2} L^{*} \psi^{*}-\beta^{*}=-\frac{1}{2} \beta^{*^{2}} / x
\end{gathered}
$$

$$
L^{*}(\cdots)=(\cdots)^{\prime \prime}+(\cdots)^{\prime} / x-(\cdots) / x^{2} .
$$

The corresponding expressions for stress resultants, stress couples, and displacements take the form

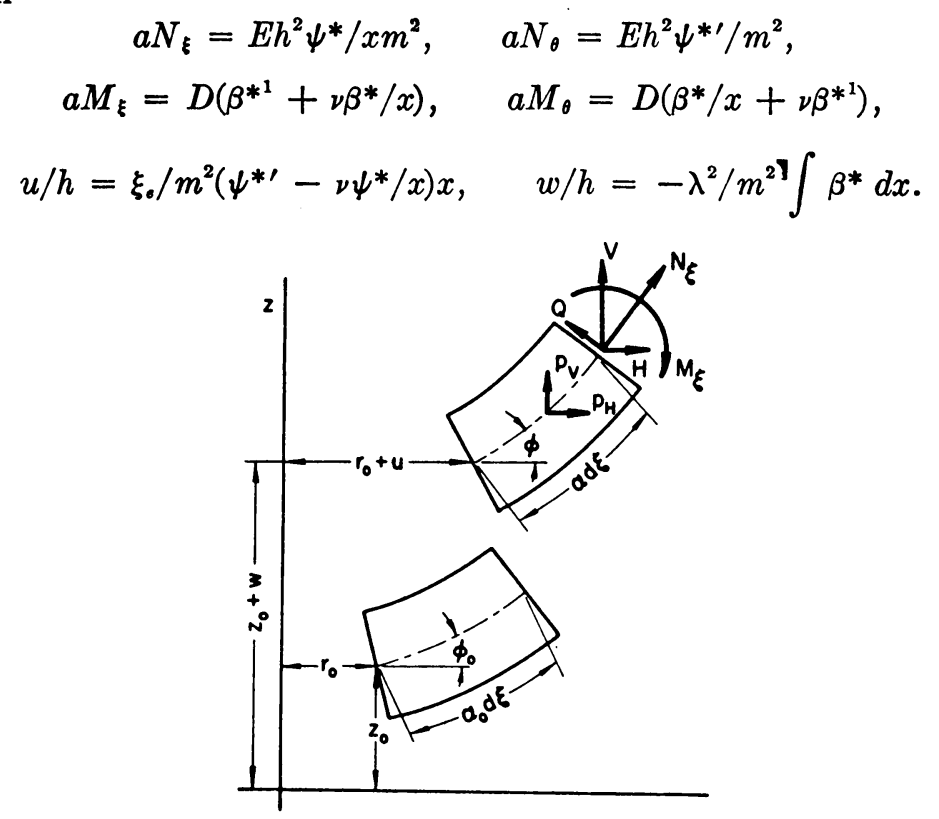

Fra. 2. Side view of element of shell in undeformed and deformed states. 
3. Statement of the problem. The edge conditions for a clamped shell segment are given by

$$
u\left(\xi_{\iota}\right)=0, \quad \beta\left(\xi_{e}\right)=0,
$$

which in non-dimensional form becomes using $(6 \mathrm{c})$

$$
\psi^{* \prime}(1)-\nu \psi^{*}(1)=0, \quad \beta^{*}(1)=0 .
$$

In addition to (8) for shells without central hole we have the condition

$$
\beta^{*}(x), \psi^{*}(x) \text { regular at } x=0 .
$$

The small finite deflections of a clamped spherical segment under uniform radial pressure are determined by solutions of the Eq. (4) subject to the conditions (8) and (9).

4. Perturbation solution. A convenient method for getting solutions of (4) is to expand $\beta^{*}, \psi^{*}$, and the inward pressure $p$ into series in powers of a certain parameter and convert (4) into a sequence of systems of linear differential equations. The perturbation parameter $W$ will take the form of a ratio of deflection to thickness as a result of conditions imposed later. Thus, we write

$$
\begin{gathered}
\beta^{*}=\sum_{l=1}^{\infty} \beta_{l} W^{l}, \quad \psi^{*}=\sum_{l=1}^{\infty} \psi_{l} W^{l}, \\
2 p=\sum_{l=1}^{\infty} p_{l} W^{l}
\end{gathered}
$$

and substitute into (4). Equating coefficients of powers of $W$ to zero leads to the sequence of linear systems

$$
\begin{gathered}
1 / \lambda^{2} L^{*} \beta_{1}+\psi_{1}=-p_{1} x, \\
1 / \lambda^{2} L^{*} \psi_{1}-\beta_{1}=0, \\
1 / \lambda^{2} L^{*} \beta_{2}+\psi_{2}=-p_{2} x+\beta_{1} \psi_{1} / x \\
1 / \lambda^{2} L^{*} \psi_{2}-\beta_{2}=-\frac{1}{2} \beta_{1}^{2} / x, \\
\vdots \\
1 / \lambda^{2} L^{*} \beta_{l}+\psi_{l}=-p_{l} x+\sum_{i+j=l} \beta_{i} \psi_{i} / x, \quad(i, j=1,2, \cdots, l-1) \\
1 / \lambda^{2} L^{*} \psi_{l}-\beta_{l}=-\frac{1}{2} \sum_{i+j=l} \beta_{i} \beta_{i} / x .
\end{gathered}
$$

The boundary conditions in terms of $\beta_{l}$ and $\psi_{l}$ become

$$
\begin{aligned}
& \beta_{l}(x), \quad \psi_{l}(x) \quad \text { regular at } \quad x=0 \\
& \beta_{l}(1)=0, \quad \psi_{l}^{\prime}(1)-\nu \psi_{l}(1)=0
\end{aligned}
$$

for all $l$.

4.1. Determination of $\beta_{1}, \psi_{1}$, and $p_{1} . \beta_{1}$ and $\psi_{1}$ are to be solutions of (11.1) satisfying the conditions (12.1). We seek solutions of (11.1) as expansions in terms of Bessel 
functions of the first kind in the form

$$
\begin{gathered}
\beta_{1}(x)=\sum_{n=1}^{\infty} a_{n}^{(1)} J_{1}\left(\lambda_{n} x\right), \\
\psi_{1}(x)=c_{1} x+\sum_{n=1}^{\infty} b_{n}^{(1)} J_{1}\left(\lambda_{n} x\right),
\end{gathered}
$$

where the $\lambda_{n}$ are defined by

$$
J_{1}\left(\lambda_{n}\right)=0 \quad(n=1,2, \cdots) .
$$

Thus, $\beta_{1}$ satisfies (12.1), and it remains to choose $c_{1}$ so that $\psi_{1}$ satisfies (12.1). From (13b), we have

$$
\psi_{1}^{\prime}(1)=c_{1}+\sum_{n=1}^{\infty} \lambda_{n} b_{n}^{(1)} J_{0}\left(\lambda_{n}\right)
$$

and

$$
\nu \psi_{1}(1)=\nu c_{1},
$$

where we have used (14) and the formula

$$
\frac{d}{d x} J_{1}\left(\lambda_{n} x\right)=\lambda_{n} J_{0}\left(\lambda_{n} x\right)-1 / x J_{1}\left(\lambda_{n} x\right) .
$$

Now $\psi_{1}$ will satisfy (12.1) if

$$
c_{1}=-\frac{1}{1-\nu} \sum_{n=1}^{\infty} \lambda_{n} b_{n}^{(1)} J_{0}\left(\lambda_{n}\right)
$$

If (13) is substituted into (11.1), it follows that

$$
\begin{aligned}
-\left(\lambda_{n} / \lambda\right)^{2} a_{n}^{(1)}+b_{n}^{(1)} & =-\left(c_{1}+p_{1}\right) \Gamma_{n}, \\
a_{n}^{(1)}+\left(\lambda_{n} / \lambda\right)^{2} b_{n}^{(1)} & =0,
\end{aligned}
$$

where we have used

$$
L^{*}\left[J_{1}\left(\lambda_{n} x\right)\right]=-\lambda_{n}^{2} J_{1}\left(\lambda_{n} x\right)
$$

and equated coefficients of $J_{1}\left(\lambda_{n} x\right)$ to zero. The $\Gamma_{n}$ are defined by

$$
x=\sum_{n=1}^{\infty} \Gamma_{n} J_{1}\left(\lambda_{n} x\right)
$$

and using

$$
\begin{aligned}
& \int_{0}^{1} x^{2} J_{1}\left(\lambda_{n} x\right) d x=-1 / \lambda_{n} J_{0}\left(\lambda_{n}\right) \\
& \int_{0}^{1} x J_{1}^{2}\left(\lambda_{n} x\right) d x=\frac{1}{2} J_{0}^{2}\left(\lambda_{n}\right)
\end{aligned}
$$

we get

$$
\Gamma_{n}=-\frac{2}{\lambda_{n} J_{0}\left(\lambda_{n}\right)}
$$


Solving (17) gives

$$
\begin{aligned}
& a_{n}^{(1)}=\frac{-2\left(c_{1}+p_{1}\right)\left(\lambda_{n} / \lambda\right)^{2}}{\lambda_{n} J_{0}\left(\lambda_{n}\right)\left[1+\left(\lambda_{n} / \lambda\right)^{4}\right]}, \\
& b_{n}^{(1)}=\frac{2\left(c_{1}+p_{1}\right)}{\lambda_{n} J_{0}\left(\lambda_{n}\right)\left[1+\left(\lambda_{n} / \lambda\right)^{4}\right]} .
\end{aligned}
$$

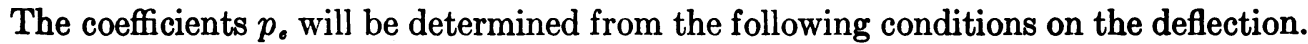
Using the expression for $w / h$ from (6c), it follows that

$$
\frac{w\left(x^{*}\right)-w(1)}{h}=-\frac{\lambda^{2}}{m^{2}} \int_{1}^{x^{*}}\left(\beta_{1} W+\beta_{2} W^{2}+\cdots\right) d x,
$$

where $x^{*}\left(0 \leq x^{*} \leq 1\right)$ will be taken at the point where the deflection is a maximum. If we impose the conditions

$$
\begin{aligned}
1 & =\lambda^{2} / m^{2} \int_{x}^{1} \beta_{1}(x) d x, \\
0 & =\int_{x *}^{1} \beta_{2}(x) d x, \\
& \vdots \\
0 & =\int_{x}^{1} \beta_{l}(x) d x,
\end{aligned}
$$

then (23) reduce to

$$
\frac{w\left(x^{*}\right)-w(1)}{h}=W
$$

which serves to define the parameter $W$. The Eq. (24) will determine the coefficients $p_{l}$ in the expansion for the inward pressure $p$.

To determine $p_{1}$, we use (13) to write (24.1) in the form

$$
\begin{aligned}
1 & =\lambda^{2} / m^{2} \int_{x^{*}}^{1} \sum_{n=1}^{\infty} a_{n}^{(1)} J_{1}\left(\lambda_{n} x\right) d x \\
& =-\frac{2\left(c_{1}+p_{1}\right) \lambda^{2}}{m^{2}} \sum_{n=1}^{\infty} \frac{\left(\lambda_{n} / \lambda\right)^{2}\left[J_{0}\left(\lambda_{n} x^{*}\right)-J_{0}\left(\lambda_{n}\right)\right]}{\lambda_{n}^{2} J_{0}\left(\lambda_{n}\right)\left[1+\left(\lambda_{n} / \lambda\right)^{4}\right]}
\end{aligned}
$$

or

$$
-2\left(c_{1}+p_{1}\right)=m^{2}\left\{\sum_{n=1}^{\infty} \frac{J_{0}\left(\lambda_{n} x^{*}\right)-J_{0}\left(\lambda_{n}\right)}{J_{0}\left(\lambda_{n}\right)\left[1+\left(\lambda_{n} / \lambda\right)^{4}\right]}\right\},
$$

where we have used the integral formula

$$
\int J_{1}\left(\lambda_{n} x\right) d x=-\frac{1}{\lambda_{n}} J_{0}\left(\lambda_{n} x\right) .
$$

Since $c_{1}$ is known from (16), $p_{1}$ is determined. 
4.2. Solution for any $\beta_{l}, \psi_{l}$, and $p_{l}$. Let

$$
\begin{gathered}
\beta_{l}(x)=\sum_{n=1}^{\infty} a_{n}^{(l)} J_{1}\left(\lambda_{n} x\right), \\
\psi_{l}(x)=c_{l} x+\sum_{n=1}^{\infty} b_{n}^{(l)} J_{1}\left(\lambda_{n} x\right),
\end{gathered}
$$

then substitution into (11.l) leads to

$$
\begin{gathered}
-\left(\lambda_{n} / \lambda\right)^{2} a_{n}^{(l)}+b_{n}^{(l)}=-\left(c_{l}+p_{l}\right) \Gamma_{n}+H_{n}^{(l)}, \\
a_{n}^{(l)}+\left(\lambda_{n} / \lambda\right)^{2} b_{n}^{l}=G_{n}^{(l)},
\end{gathered}
$$

where the $G_{n}^{(l)}, H_{n}^{(l)}$ are defined by

$$
\begin{aligned}
& G_{n}^{(l)}=\frac{1}{2} \sum_{i=1}^{\infty} \sum_{i=1}^{\infty} C_{n}^{i j}\left(\sum_{s+t=l} a_{i}^{(s)} a_{i}^{(t)}\right), \quad(s, t=1,2, \cdots, l-1) \\
& H_{n}^{(l)}=\sum_{s+t=l} c_{s} a_{n}^{(t)}+\sum_{i=1}^{\infty} \sum_{i=1}^{\infty} C_{n}^{i j}\left(\sum_{s+t=l} a_{i}^{(s)} b_{i}^{(t)}\right)
\end{aligned}
$$

and the $C_{n}^{i j}$ defined by

$$
1 / x J_{1}\left(\lambda_{i} x\right) J_{1}\left(\lambda_{i} x\right)=\sum_{n=1}^{\infty} C_{n}^{i i} J_{1}\left(\lambda_{n} x\right)
$$

The $G_{n}^{(l)}$ and $H_{n}^{(l)}$ are known from the $1,2, \cdots, l-1$ stages of the computation. Solving (29), gives

$$
\begin{gathered}
a_{n}^{(l)}=\left\{-\frac{2\left(c_{l}+p_{l}\right)\left(\lambda_{n} / \lambda\right)^{2}}{\lambda_{n} J_{0}\left(\lambda_{n}\right)}-\left(\frac{\lambda_{n}}{\lambda}\right)^{2} H_{n}^{(l)}+G_{n}^{(l)}\right\}\left[1+\left(\lambda_{n} / \lambda\right)^{4}\right]^{-1} \\
b_{n}^{(l)}=\left\{\frac{2\left(c_{l}+p_{l}\right)}{\lambda_{n} J_{0}\left(\lambda_{n}\right)}+H_{n}^{(l)}+\left(\lambda_{n} / \lambda\right)^{2} G_{n}^{(l)}\right\}\left[1+\left(\lambda_{n} / \lambda\right)^{4}\right]^{-1}
\end{gathered}
$$

and for the lth stage (16) becomes

$$
c_{l}=-\frac{1}{1-\nu} \sum_{n=1}^{\infty} \lambda_{n} b_{n}^{(l)} J_{0}\left(\lambda_{n}\right)
$$

which forces $\psi_{l}$ to satisfy the boundary condition (12.l). The condition (24.l) leads to an equation for the $l$ th stage similar to (26) for the first in the form

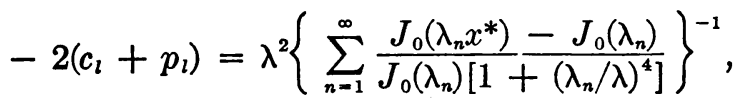

$$
\begin{aligned}
& \cdot\left\{\sum_{n=1}^{\infty} \frac{\left[\left(\lambda_{n} / \lambda\right)^{2} H_{n}^{(l)}-G_{n}^{(l)}\right]\left[J_{0}\left(\lambda_{n} x^{*}\right)-J_{0}\left(\lambda_{n}\right)\right]}{\lambda_{n}\left[1+\left(\lambda_{n} / \lambda\right)^{4}\right]}\right\} \text {. }
\end{aligned}
$$

Therefore, $c_{l}$ and $p_{l}$ are determined; and the $a_{n}^{(l)}$ and $b_{n}^{(l)}$ are completely determined.

Starting with $a_{n}^{(1)}, b_{n}^{(1)}, c_{1}$, and $p_{1}$; the $G_{n}^{(2)}$ and $H_{n}^{(2)}$ can be computed and then $a_{n}^{(2)}, b_{n}^{(2)}, c_{2}$, and $p_{2}$. Similarly, $G_{n}^{(l)}$ and $H_{n}^{(l)}$ are computed leading to $a_{n}^{(l)}, b_{n}^{(l)}, c_{l}$, and $p_{l}$. This can be continued to obtain any number of terms in the series for $\beta^{*}, \psi^{*}$, and $2 p$. Stresses and displacements can be computed from the Eq. (6). The buckling pressure 
is determined from

$$
2 p=\sum_{l=1}^{\infty} p_{l} W^{l}
$$

by the condition

$$
d p / d W=0
$$

5. Numerical solutions. This section involves numerical calculations using the integration procedure set up in the previous section to obtain new results for the finite deflections of a clamped spherical shell segment under uniform pressure. The perturbation of the non-linear equations is carried out far enough to determine the deflection curves as a function of the pressure for maximum deflections up to about one thickness of the shell. For the case of inward pressure, the buckling phenomenon is observed in this range of deflections and buckling pressures are found using the "classical criterion" for a range of the central height to thickness ratio from about 1 to 35. (See Figs. 3 and 4).

In the numerical computation for this problem or for other cases of loading and edge restraint involving shallow spherical shells, it is necessary to compute the expansion coefficients, $c_{n}^{i j}$, which enter the computation from the non-linear terms. These were computed directly from the definition

$$
C_{n}^{\prime i}=\left[\frac{1}{2} J_{0}^{2}\left(\lambda_{n}\right)\right]^{-1} \int_{0}^{1} J_{1}\left(\lambda_{i} x\right) J_{1}\left(\lambda_{j} x\right) J_{1}\left(\lambda_{n} x\right) d x
$$

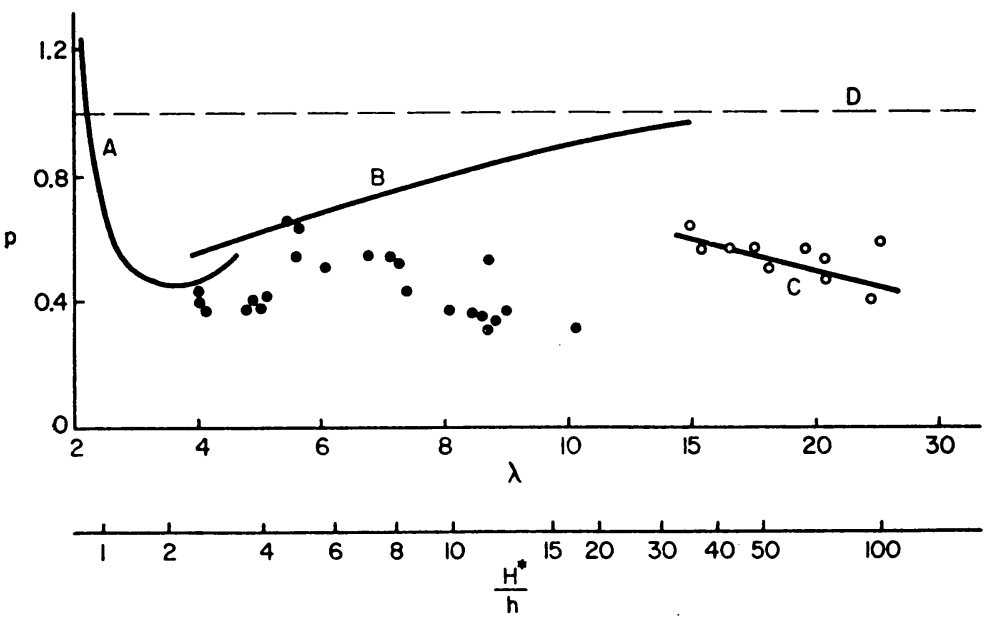

Fra. 3. Known theoretical and experimental results are compared. A represents the theory in [5], $B$ the theory in this paper, $C$ the theory in [12], the solid dots the experiments in [5], the hollow dots the experiments in [12], and $D$ the classical buckling load for a complete sphere given here for reference.

using Simpson's rule with one hundred values of the integrand. The results obtained by machine computation are given in Table 1. It is shown in [1] that these coefficients can be used to solve a variety of other problems involving other conditions of loading and edge restraint.

In determining the buckling pressures given in Fig. 3, terms were computed in the perturbation series used in finding the pressure-maximum deflection curves until it was 
seen that the higher order terms were no longer substantially affecting the curves in the neighborhood of the point where $d p / d W=0$. The linear solution checks the linear solution given in [5] which is the same as the one given by Reissner in [7]. The second

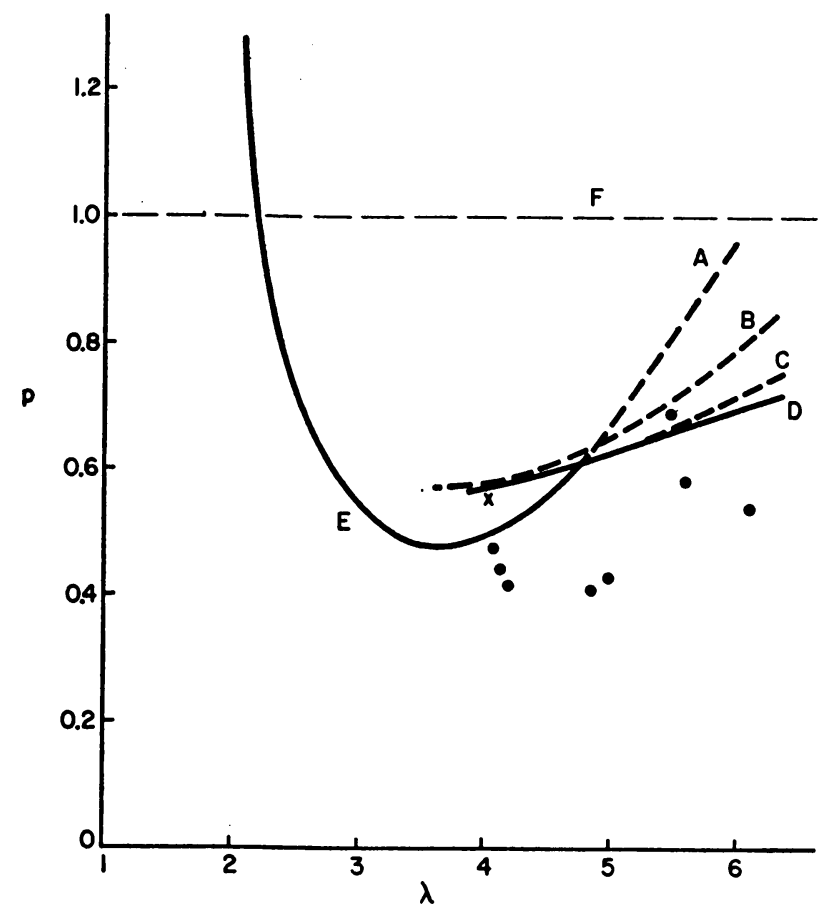

Frg. 4. The curves $A, B, C$, and $D$ represent results using two, three, four, and five terms respectively in the perturbation series of this paper. $E$ and the cross are computed in [5] using two and four terms respectively in a perturbation series while the solid dots represent the experimental results reported in [5]. $F$ represents the buckling pressure for a complete sphere (linear theory) and is given here for reference.

TABLE 1a. Numerical values for the expansion coefficients $C_{1}^{i j}$ and $C_{2}^{i j}$.

\begin{tabular}{|c|c|c|c|c|c|c|c|}
\hline$C_{1}^{i j} \times 101$ & 2 & 3 & 4 & 5 & 6 & 7 & 8 \\
\hline 10.123 & 3.0232 & -0.20513 & 0.067211 & -0.029812 & 0.015362 & -0.0094689 & 0.0060496 \\
\hline 2 & 5.9445 & 1.9771 & -0.15236 & 0.054438 & -0.025949 & 0.014360 & -0.0089100 \\
\hline 3 & & 4.1592 & 1.4594 & -0.11913 & 0.044558 & -0.029812 & 0.012264 \\
\hline 4 & & & 3.1925 & 1.1564 & -0.097467 & 0.037596 & -0.032196 \\
\hline$i 5$ & & & & 2.5891 & 0.95663 & -0.082360 & 0.032196 \\
\hline 6 & & & & & 2.1770 & 0.81565 & -0.071173 \\
\hline 7 & & & & & & 1.8778 & 0.71087 \\
\hline 8 & & & & & & & 1.6506 \\
\hline \multicolumn{8}{|l|}{$C_{2}^{i j} \times 10$} \\
\hline 5.4449 & 10.706 & 3.5608 & -0.27440 & 0.098043 & -0.046734 & 0.025862 & -0.016047 \\
\hline 2 & 8.1792 & 7.4106 & 2.4816 & -0.21304 & 0.082514 & -0.042530 & 0.023878 \\
\hline 3 & & 6.1991 & 5.5398 & 1.8982 & -0.17083 & 0.069546 & -0.036298 \\
\hline 4 & & & 4.88463 & 4.4079 & 1.5366 & -0.14285 & 0.058814 \\
\hline i5 & & & & 4.0093 & 3.6553 & 1.2906 & -0.12160 \\
\hline 6 & & & & & 3.3932 & 3.1208 & 1.1123 \\
\hline 7 & & & & & & 2.9386 & 2.7218 \\
\hline 8 & & & & & & & 2.5901 \\
\hline
\end{tabular}


TABLE 1b. Numerical values for the expansion coefficients $C_{3}^{i j}$ and $C_{4}^{i j}$.

\begin{tabular}{|c|c|c|c|c|c|c|c|}
\hline$C_{3}^{i f} \times 10$ & 2 & 3 & 4 & i5 & 6 & 7 & 8 \\
\hline-0.53368 & 5.1437 & 10.821 & 3.7968 & -0.30992 & 0.11592 & -0.05795 & 0.031905 \\
\hline 2 & 10.705 & 8.9547 & 8.0023 & 2.7420 & -0.24677 & 0.10046 & -0.052433 \\
\hline 3 & & 9.0110 & 7.3074 & 6.2026 & 2.1426 & -0.20310 & 0.085728 \\
\hline 4 & & & 7.2969 & 5.9928 & 5.0455 & 1.7617 & -0.16981 \\
\hline i5 & & & & 6.0597 & 5.0423 & 4.2489 & 1.4948 \\
\hline 6 & & & & & 5.1395 & 4.3403 & 3.6684 \\
\hline 7 & & & & & & 4.4845 & 3.8044 \\
\hline 8 & & & & & & & 3.9618 \\
\hline \multicolumn{8}{|l|}{$C_{4}^{i j} \times 10$} \\
\hline 0.22866 & -0.51834 & 4.9651 & 10.861 & 3.9340 & -0.33159 & 0.12791 & -0.063310 \\
\hline 2 & 4.6872 & 10.465 & 9.2271 & 8.3266 & 2.9027 & -0.26985 & 0.11110 \\
\hline 3 & & 9.5559 & 9.5422 & 7.8368 & 6.5981 & 2.3038 & -0.22206 \\
\hline 4 & & & 8.4625 & 8.0664 & 6.5892 & 5.4518 & 1.9135 \\
\hline i5 & & & & 7.2569 & 6.7884 & 5.6391 & 4.6415 \\
\hline 6 & & & & & 6.2774 & 5.9621 & 4.9127 \\
\hline 7 & & & & & & 5.5059 & 5.2500 \\
\hline 8 & & & & & & & 4.8913 \\
\hline
\end{tabular}

TABLE 1c. Numerical values for the expansion coefficients $C_{5}^{i j}$ and $C_{6}^{i j}$.

\begin{tabular}{|c|c|c|c|c|c|c|c|c|}
\hline & $+1 \quad 1$ & 2 & 3 & 4 & ${ }^{i} 5$ & 6 & 7 & 8 \\
\hline 1 & -0.12529 & 0.22878 & -0.50064 & 4.8597 & 10.881 & 4.0204 & -0.34613 & 0.13531 \\
\hline 2 & & -0.49712 & 4.4294 & 10.286 & 9.3556 & 8.5295 & 3.0115 & -0.28374 \\
\hline 3 & & & 10.020 & 9.6808 & 9.7889 & 8.1454 & 6.8637 & 2.4148 \\
\hline 4 & & & & 9.9644 & 8.9645 & 8.4968 & 6.9660 & 5.7337 \\
\hline 5 & & & & & 8.8285 & 7.9038 & 7.3757 & 6.0342 \\
\hline 6 & & & & & & 7.7507 & 6.9680 & 6.4774 \\
\hline 7 & & & & & & & 6.8521 & 6.1939 \\
\hline 8 & & & & & & & & 6.1189 \\
\hline$C$ & $0^{+1}$ & & & & & & & \\
\hline 1 & 0.07686 & -0.12983 & 0.2293 & -0.48764 & 4.7862 & 10.892 & 4.0808 & -0.35609 \\
\hline 2 & & 0.22922 & -0.47456 & 4.2687 & 10.154 & 9.4264 & 8.6695 & 3.0890 \\
\hline 3 & & & 4.1205 & 9.7031 & 9.6969 & 9.9222 & 8.3468 & 7.0547 \\
\hline 4 & & & & 9.6901 & 10.115 & 9.2316 & 8.7678 & 7.2246 \\
\hline$i 5$ & & & & & 9.4093 & 9.2271 & 8.2952 & 7.112 \\
\hline 6 & & & & & & 8.5468 & 8.2610 & 7.4116 \\
\hline 7 & & & & & & & 7.6093 & 7.4078 \\
\hline 8 & & & & & & & & 6.9348 \\
\hline
\end{tabular}

TABLE 1d. Numerical values of the expansion coefficients $C_{7}^{i j}$ and $C_{8}^{i j}$.

$\begin{array}{ccccccccc}C_{7}^{i j} \times 10^{+1} 1 & 1 & 2 & 3 & 4 & i 5 & 6 & 7 & 8 \\ 1 & -0.054954 & 0.083337 & -0.12927 & 0.21819 & -0.47798 & 4.7337 & 10.898 & 4.1255 \\ 2 & & -0.13705 & 0.22411 & -0.46033 & 4.1587 & 10.057 & 9.4692 & 8.7706 \\ 3 & & & -0.45308 & 3.9300 & 9.4784 & 9.6822 & 10.004 & 8.4868 \\ 4 & & & & 9.3001 & 9.6196 & 10.171 & 9.3924 & 8.9559 \\ i 5 & & & & & 10.185 & 9.6224 & 9.4624 & 8.5534 \\ 6 & & & & & & 9.5827 & 8.9196 & 8.5929 \\ 7 & & & & & & & 8.7609 & 8.1406 \\ 8 & & & & & & & & 7.9766\end{array}$




\begin{tabular}{|c|c|c|c|c|c|c|c|c|}
\hline \multicolumn{9}{|c|}{$C_{8}^{i j} \times 10^{+1}$} \\
\hline 1 & 0.039952 & -0.058843 & 0.080990 & -0.12290 & 0.21263 & -0.47003 & 4.6946 & 10.901 \\
\hline 2 & & 0.087558 & -0.13310 & 0.21567 & -0.44588 & 4.07865 & 9.9804 & 9.4976 \\
\hline 3 & & & 0.21762 & -0.43106 & 3.7946 & 9.3122 & 9.6574 & 10.057 \\
\hline 4 & & & & 3.7145 & 9.0100 & 9.5364 & 10.191 & 9.4949 \\
\hline 5 & & & & & 9.4823 & 10.178 & 9.7333 & 9.6154 \\
\hline 6 & & & & & & 9.7833 & 9.7782 & 9.1540 \\
\hline 7 & & & & & & & 9.2635 & 9.0768 \\
\hline 8 & & & & & & & & 8.5853 \\
\hline
\end{tabular}

order solution checks with that given in [5] for $3 \leq \lambda \leq 5$ where the maximum deflection is at the center.

The Massachusetts Institute of Technology digital computer, Whirlwind I, was used extensively to reduce the computation time. The programs used for the machine were checked by independent calculations using a desk calculator.

In Figs. 3 and 4, the stability limits found in this paper are compared with known experimental and theoretical results. It is seen that the theoretical curve given in [5] based on two terms of a perturbation series is subject to considerable correction when more terms in the series are computed. In particular, the minimum value of $H^{*} / h$ for which buckling occurs must be revised upward from the value of about 0.67 given in [5] to 2.2 found in this paper. Also, it is seen that the experimental results depart from the theoretical results of this investigation as $H^{*} / h$ increases. As indicated in the references, this is probably due to the fact that the shell, under disturbances during the testing, jumps to a nearby buckles state before reaching the buckling pressure predicted by the theory in this paper which allows only continuous load-deflection processes.

6. Acknowledgments. The author would like to take this opportunity to thank his thesis supervisor, Professor Eric Reissner, for his help and guidance during the course of this investigation.

The author would also like to thank the members of the M.I.T. Committee for Machine Computation for authorizing computer time for this problem.

\section{REFERENCES}

1. R. R. Archer, On the post buckling behavior of thin spherical shells, Ph.D. thesis, M.I.T. Math. Dept., 1956

2. C. B. Biezeno, Über die Bestimmung der "Durchschlagkraft" einer schwachgekrümmten, kreisförmigen Platte, Z.A.M.M. 15, 10-22 (1935)

3. V. I. Feodosev, On the stability of a spherical shell under the action of an external uniform pressure, (in Russian), Prikl. Mat. Mek. (1) 18, 35-42 (1954)

4. K. O. Friedrichs, On the minimum buckling load for spherical shells, Theo. von Karman anniversary volume, California Institute of Technology, Pasadena, 1941, pp. 258-272

5. A. Kaplan and Y. C. Fung, A non-linear theory of bending and buckling of thin shallow spherical shells, Natl. Advisory Comm. Aeronaut., Tech. Notes 3212, (1954)

6. H. M. Mushtari and R. G. Surkin, On the non-linear stability theory of elastic equilibrium of a thin spherical shell under the influence of uniformly distributed normal external pressure, (in Russian), Prikl. Mat. Mek. (6) 14, 573-586 (1950)

7. E. Reissner, Stresses and small displacements of shallow spherical shells II, J. Math. and Phys. 25, 279-300 (1947)

8. E. Reissner, On axisymmetrical deformation of thin shells of revolution, Proc. Symp. on Appl. Math., Am. Math. Soc. 3, 27-52 (1950)

9. R. M. Simons, On the non-linear theory of thin spherical shells, Ph.D. thesis, M.I.T. Math. Dept., 1955 
10. S. Timoshenko, Theory of elastic stability, McGraw-Hill Book Co., Inc., New York, 1936

11. S. Timoshenko, Theory of plates and shells, McGraw-Hill Book Co., Inc., New York, 1940

12. H. S. Tsien, $A$ theory for the buckling of thin shells, J. Aeronaut. Sci. 9, (1940)

13. H. S. Tsien, Lower buckling load in the non-linear buckling theory for thin shells, Quart. Appl. Math. 5, 236 (1947)

14. M. Uemura and Y. Yoshimura, The buckling of spherical shells by external pressure II, (in Japanese), Repts. Inst. Sci. and Technol., Tokyo (6) 6, 367-371 (1950)

15. Th. von Karman and H. S. Tsien, The buckling of spherical shells by external pressure, J. Aeronaut. Sci. (2) 7, 43-50 (1939)

16. Y. Yoshimura and M. Uemura, The buckling of spherical shells due to external pressure I, (in Japanese), Repts, Inst. Sci. and Technol., Tokyo 3, 316-322 (1949) 